\title{
Satellite Stereo Image Processing for 3D Topographic Mapping
}

\author{
Jaehong $\mathrm{Oh}^{\mathrm{a},} *$, Changno Lee ${ }^{\mathrm{b}}$ \\ ${ }^{a}$ Korea Maritime and Ocean University, jhoh@kmou.ac.kr \\ ${ }^{b}$ Seoul National University of Science and Technology, changno@seoultech.ac.kr \\ * Corresponding author
}

Keywords: Satellite, Stereo Images, Epipolar, Topographic Map

\begin{abstract}
:
The large scale topographic map generation is mostly carried out using the aerial images while the high resolution satellite data are gaining popularity because of its large swath width that enables the efficient and economic mapping even over inaccessible areas. To use the satellite data for the 3D topographic mapping, the data should be acquired in the stereo mode and they requires to be aligned for the epipolar geometry. The accurate epipolar image resampling aligns stereo images to enable a stereo display on the computer monitor where human operators can easily identify and extract 3D features, such as points of interest, contours, building layers, and roads. The pushbroom type camera, which is used by most high-resolution satellites, has quite different epipolar geometry than the frame type of aerial or terrestrial cameras. Therefore, the piecewise approach should be used instead of the fundamental matrix approach that is the standard method for the frame type cameras. Regarding the sensor model, most high-resolution satellite data use RPCs (Rational Polynomial Coefficients) of RFM (Rational Function Model) while the frame type camera use the collinearity equation. But RPCs from the satellite image providers have not been accurate enough such that GCPs (Ground Control Points) are often required for improving the RPCs accuracy. The GCPs acquisition is not an easy task over the inaccessible areas and the positional accuracy of the old geospatial data such as orthoimages and traditional maps is relatively low. Fortunately, the positional accuracy of RPCs increases than the past such as up to several meters. The major problem to achieve high accuracy of stereo geometry is to ensure that the consistency between the stereo data is less than one-pixel level. Therefore, in this study, we first utilized the relative orientation method to improve the precision between the stereo data without using any GCP. The tie points are extracted using the robust stereo matching and they are used to generate quasi-GCPs by the space intersection based on the RPCs. The quasi-GCPs are projected back to the stereo data to model and remove the inconsistency in the image space. The improved RPCs were used to accurately align the stereo data for map production.
\end{abstract}

Acknowledgement: This research was supported by a grant(19SIUE-B148326-02) from CAS 500-1/2 Image Acquisition and Utilization Technology Development Program funded by Ministry of Land, Infrastructure and Transport of Korean government 